\title{
Langerhans' cell histiocytosis of the eyelid
}

\author{
Christos Daras, Wayne Grayson, Ismael Mayet, Sarya Saks, Clive Novis, Neville H Welsh
}

\section{Department of \\ Ophthalmology, \\ University of the \\ Witwatersrand, St John \\ Eye Hospital, \\ Johannesburg, \\ South Africa \\ $\mathrm{N} \mathrm{H}$ Welsh \\ C Daras \\ I Mayet \\ S Saks \\ C Novis}

\section{Department of}

Anatomical Pathology,

University of the

Witwaterstrand and the

South African Institute

for Medical Research,

Johannesburg,

South Africa

W Grayson

Correspondence to:

Dr C Daras, Department of

Ophthalmology, University of

the Witwaterstrand, 7 York

Road, Parktown 2193,

South Africa.

Accepted for publication

19 August 1994
We present an unusual case of a Langerhans' cell histiocytosis $(\mathrm{LCH})$ presenting as a lid mass with secondary cervical lymphadenopathy on the ipsilateral side.

Proliferative disorders of histiocytes comprise a wide spectrum of conditions ranging from solitary lesions that usually exhibit benign clinical behaviour to widely disseminated lesions that may exhibit a malignant course. The term Langerhans' cell histiocytosis is currently used to include eosinophilic granuloma, HandSchuller-Christian disease, and Letterer-Siwe disease. All these entities have very similar histopathological features although their clinical manifestations vary and often overlap. Orbital involvement may vary, but the most common orbital lesion is a solitary osseous lesion formerly referred to as eosinophilic granuloma. ${ }^{1}$ Furthermore, it has been been suggested that the majority of orbital lesions arise from bone. ${ }^{2}$ Tosaka $^{3}$ has reported an isolated histiocytosis $\mathrm{X}$ lesion in an eyelid in a 33-year-old man. We have been unable to find any reports of an LCH lesion presenting as an isolated eyelid mass in a child.

\section{Case report}

A 3-year-old black girl presented for evaluation of a right upper lid mass that was noticed 4 weeks before presentation. There was no history of any medical disorder or of any trauma. The family history was non-contributory.

On examination a large mass $3 \mathrm{~cm}$ long by $2 \mathrm{~cm}$ high was felt over the upper lid. The mass was not mobile and extended up to the lid margin. There were no signs of infection or any ulceration of the overlying skin. General examination revealed no lymphadenopathy or any organ-

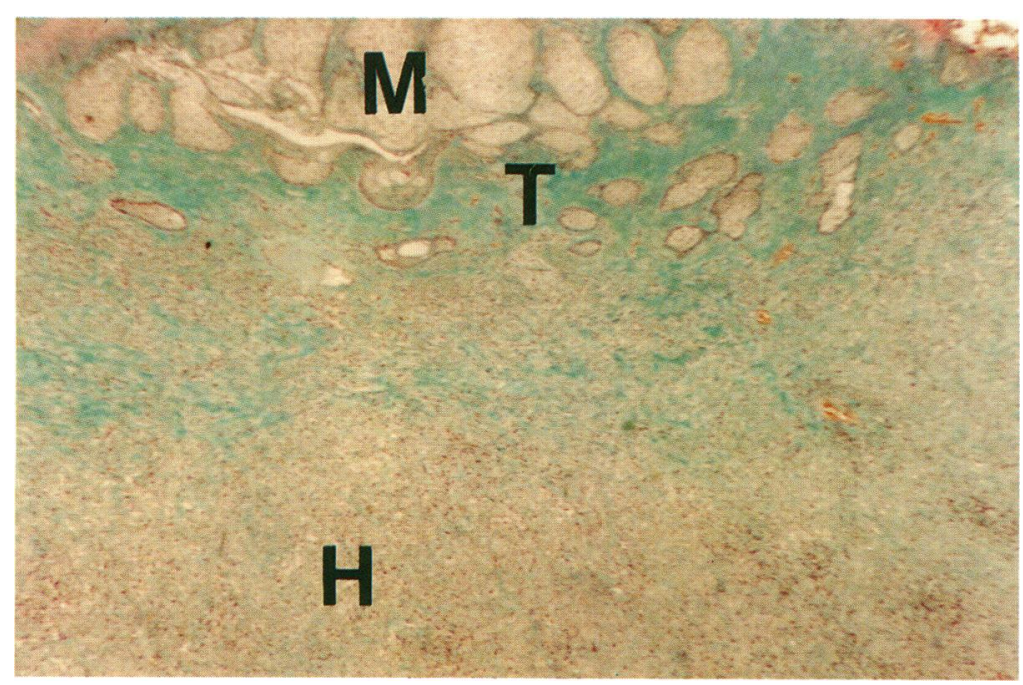

Figure 1 Low power micrograph of trichome stained section of upper eyelid mass. The abnormal population of Langerhans' histiocytes $(H)$ is seen abutting on the fibrous tarsal plate $(T)$ and subjacent Meibomian glands $(M)$ at the deep aspect of the specimen (Masson, $\times 43)$. omegaly. $x$ Rays of the lungs and the skull revealed no abnormalities.

The tumour was excised under general anaesthesia. A broad solid pedunculated mass was found adherent to the anterior tarsal plate and extended up to the lid margin. The tarsal plate was thickened. The specimen was submitted for histological examination. Right sided shotty cervical lymphadenopathy was subsequently noticed 7 days after the initial presentation.

Histological examination revealed the presence of a highly cellular lesion covered by benign squamous epithelium (Fig 1). The lesion was characterised by a sheet-like proliferation of tightly packed differentiated histiocytes, including occasional binucleated and multinucleated forms. The histiocytic cells had relatively indistinct intercellular borders and abundant pale eosinophilic cytoplasm (Fig 2a). Their pale vesicular nuclei were oval to reniform. Several nuclei appeared indented and convoluted, some assuming a horseshoe-like configuration. Occasional small eosinophilic nucleoli were discernible. A few scattered mitotic figures were identified. There was a prominent background infiltrate of eosinophilic leucocytes, with several small eosinophilic 'micro-abscesses'.

In the presence of adequate and appropriate controls, immunohistochemical stains for S-100 protein proved strongly positive within the cytoplasm of the histiocytic cells, confirming their differentiated nature (Fig $2 b$ ).

Transmission electron microscopy confirmed the presence of large cells with bulky cytoplasm and irregular, grooved, deeply indented nuclei with peripherally condensed chromatin and discernible nucleoli. Detailed examination of the region of the cell membrane revealed numerous scattered pentalaminar rod-shaped structures of varying length which were continuous with the cell membrane. One or two of these so-called Birbeck granules exhibited terminal expansions where one end of the granule was dilated to form a saccule which was bounded by the outer membrane of the rod, resulting in a tennis racquet shape (Fig 3). The histopathological features were those of Langerhans' cells histiocytosis.

A fine needle aspirate of the cervical lymph nodes confirmed the presence of an abnormal histiocyte population, morphologically identical to that found in the eyelid mass.

\section{Comment}

Langerhans' cell histiocytosis has distinctive pathological characteristics which include the presence of Langerhans' cells as part of the lesion. ${ }^{+5}$ The presence of Birbeck granules in the 


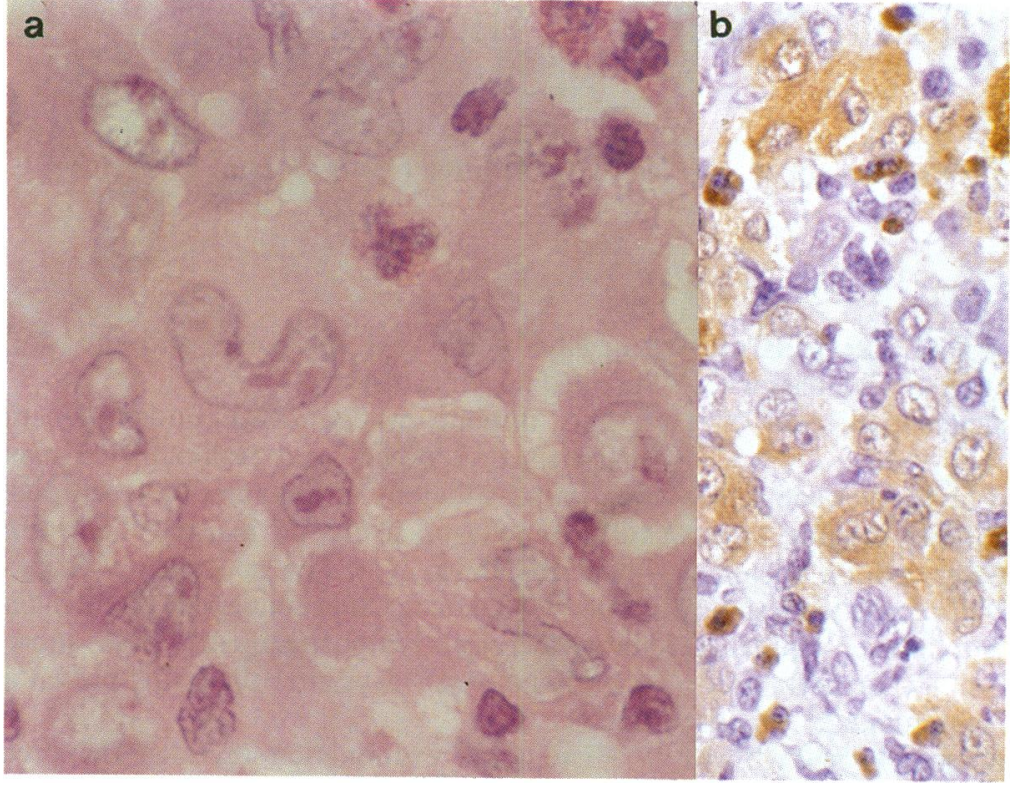

Figure 2 (a) Sheet-like projection of differentiated histiocytic cells, some with large reniform nuclei. Note the background of eosinophilic leucocytes (haematoxylin and eosin, $\times 777)$. (b) Strongly positive immunoreactivity for $S-100$ protein within the cytoplasm of the histiocytic cells (immunoperoxidase, $\times 300$ ). lesional cells or the demonstration of T6 antigenic determinants on the surface of lesional cells, is required for a definitive diagnosis of LCH to be made. ${ }^{45}$

The patient in this case presented with a solitary lesion manifesting as a lid mass (with subsequent lymphadenopathy). with no visceral or osseous involvement. The mass was confined to the soft tissue of the eyelid and was closely related to the overlying squamous epithelium. In addition, the 'classic' clinical characteristics of the histiocytosis X diseases were not evident.

Eight cases of solitary lesions in soft tissues have been reported under the designation of congenital self-healing reticulohistiocytosis (CSHR) or Hashimoto-Pritzker disease. ${ }^{6}$ However, our patient's age did not fit the criteria for CSHR; there was no spontaneous involution of the lesion; and there was a progression of the disease with the appearance of the lymphadenopathy. ${ }^{5}$

We therefore conclude that the case presented here be classified as a solitary nodular LCH according to the criteria of the writing group of the Histiocyte Society. ${ }^{4}$ However, owing to the proposed reactive pathogenesis of this disease, ${ }^{5}$ we propose that the appearance of the ipsilateral cervical lymphadenopathy be seen as a secondary involvement of the lymph nodes that drain that part of the eyelid.

The authors thank Mrs Glenys King of the electron microscopy unit of the SAIMR for her assistance.

1 Shields JA. Histiocytic tumours and pseudotumours. In: Diagnosis and management of orbital tumours, Philadelphia: Saunders, 1989: 378-88.

2 Moore AT, Pritchard J, Taylor DS. Histiocytosis X: an ophthalmological review. Brf Ophthalmol 1985; 69: 7-14.

3 Tosaka Y. A case of localised histiocytosis X of the eyelid. Nippon Ganka Gakkai Zasshi 1989; 93: 103-8.

4 Writing group of the Histiocyte Society. Histiocytosis in children. Lancet 1987; i: 208-9.

5 Malone M. The histiocytoses of childhood. Histopathology 1991; 19: 105-19.

6 Ikeda M, Yamamoto Y, Kitagawa N, Kodama H, Moriki T, Hiroi $M$. Solitary nodular Langerhans cell histiocytosis. $B r \mathcal{F}$ Dermatol 1993; 128: 220-2.
Figure 3 Electron differentiated histiocyte from case of Langerhans' cell histiocytosis showing highly convoluted nuclear membrane, and a Birbeck granule (arrow) arising from the cell membrane (uranyl acetate and lead citrate, $\times 16800)$. Inset: detail of pentalaminar Birbeck granule with dilated terminal saccule (uranyl acetate and lead citrate, $\times 28000)$.

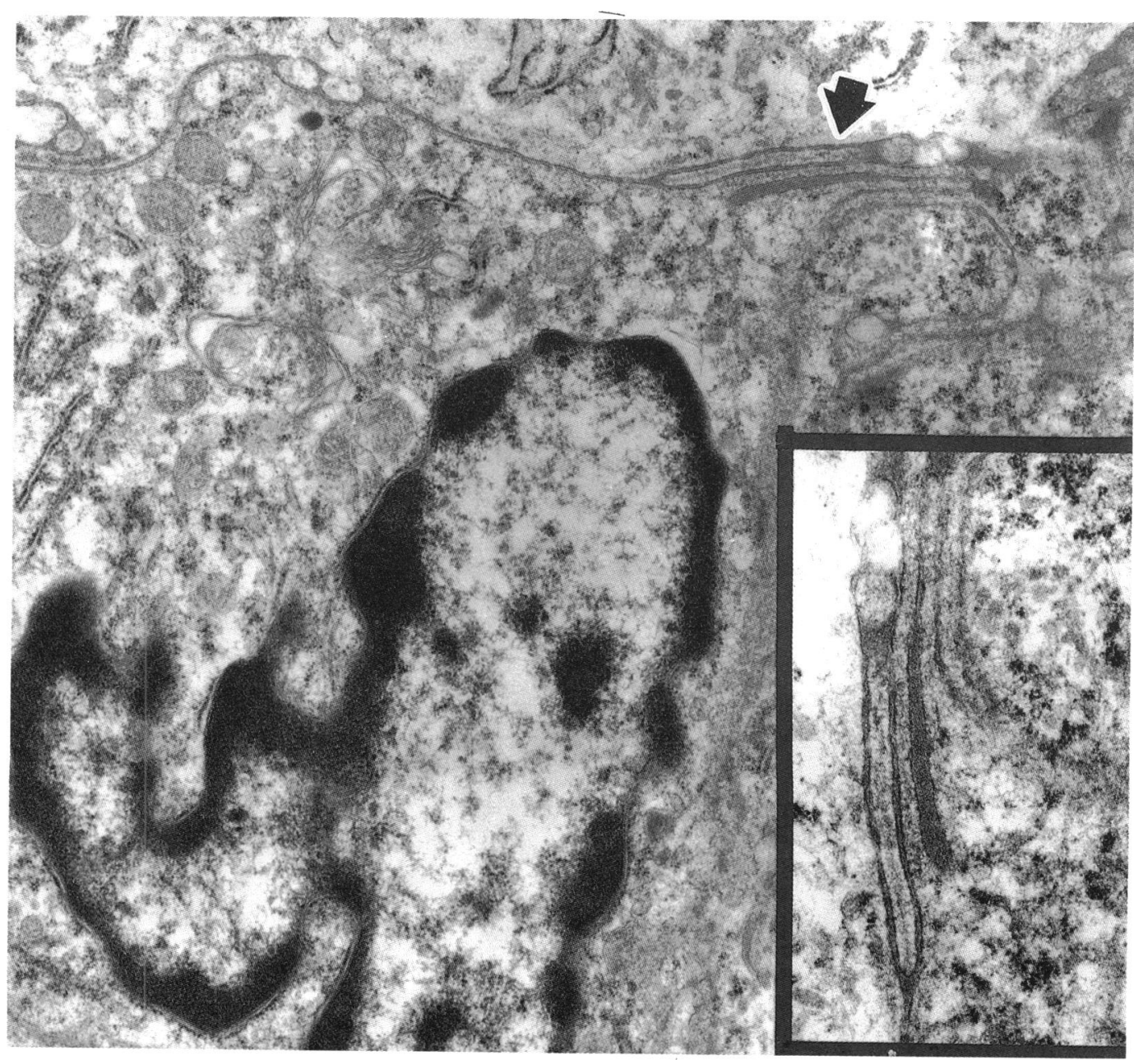

